\title{
DESENVOLVIMENTO DE EMULSÃO DERMATOCOSMÉTICA CONTENDO MANTEIGA DE MANGA (Mangifera indica L.) Anacardiaceae.
}

\section{COMPARATIVE SENSORY EVALUATION OF DERMOFORMULAÇÃO CONTAINING BUTTER MANGO (Mangifera indica)}

\author{
Dallarmi, L. ; ;iguel, M.D. ${ }^{1,2}$; Cansian, F.C. \\ ${ }^{1}$ Laboratório de Farmacotécnica, Departamento de Farmácia, Universidade Federal do \\ Paraná, Curitiba-PR, Brasil.Email: fecolombi@hotmail.com \\ ${ }^{2}$ Professora da Disciplina de Farmacotécnica da Universidade Federal do Paraná
}

\section{RESUMO:}

Ao longo dos últimos anos assistimos um aumento no uso e valorização de prescrições de fórmulas magistrais envolvendo dermocosméticos, constituídos geralmente por substâncias que auxiliam a pele a manter-se em perfeitas condições, ou seja, hidratada e saudável. Dentre estas substâncias destacam-se as de origem vegetal, utilizadas principalmente para o tratamento de pele ressecada, como por exemplo, a manteiga de manga (Mangifera indica), pertencente à família Anacardiaceae. A substância é extraída do caroço do fruto da manga, composta quimicamente por uma mistura de ácidos graxos que justifica suas propriedades emolientes e regeneradoras da epiderme, o que levou ao desenvolvimento de uma emulsão base para sua incorporação. Foram analisadas as propriedades físicas-químicas incluindo a avaliação sensorial desta emulsão, confirmando o potencial hidratante da manteiga de manga, uma vez que $97 \%$ dos voluntários sentiram sua pele protegida após o uso.

\section{Palavras Chave: Avaliação Sensorial, Dermoformulação, Manteiga de Manga.}

\section{ABSTRACT:}

Over the past few years we've been seeing a growing trend to use and recovery prescription formulas masterful in dealing with skin care. These products are usually made of substances that help to keep skin in perfect condition, ie, hydrated and healthy. Among such substances has been seconded from the plant for the treatment of dry skin. In this context, is the mango butter (Mangifera indica), the family of Anacardiaceae, a raw material extracted from the seed of the fruit of mango, chemically composed of a mixture of fatty acids that justifies its emollient properties and regenerating the epidermis. This raw material led to the development of an emulsion base for its incorporation. In the sensory evaluation of the emulsion, it is confirmed the potential of moisturizing mango butter, since $97 \%$ of volunteers felt their skin protected after use.

Key-words: Sensory Evaluation, Dermal Formulation, Mango Butter 


\section{INTRODUÇÃO}

Nas últimas décadas a preferência pelos produtos manipulados vem aumentando significativamente. A farmácia magistral atende diversas especialidades e segundo Miguel, et al (2002), 18 \% das formulações são emulsões dermatológicas e estéticas.

A indústria farmacêutica tem desenvolvido inúmeros ativos e bases dermatológicas de origem vegetal que quando incorporados a formas farmacêuticas podem dar origem a medicamentos, nutracêuticos e cosméticos (Bergwein et al, 1968).

As gorduras vegetais tem sido muito utilizadas não apenas por serem bons emolientes como também por oferecerem interessantes propriedades bioativas (Mandawgade \& Patravale, 2008). Pertencente à família Anacardiaceae, a manteiga de manga (Mangifera indica L.) é extraída do caroço da manga, apresentando em seu conteúdo, apreciável teor de tocoferol, fitoesteróis e triterpenos, tornando-a um ingrediente cosmético funcional com grande potencial (Bhattacharya \& Shukla, 2002).

Por ser uma substância pouco explorada na manipulação cosmecêutica e com grandes propriedades, o presente estudo propõe o desenvolvimento de um produto dermocosmético, utilizando a manteiga de manga como ativo principal em uma emulsão nutritiva e hidratante.

\section{MATERIAL E MÉTODOS}

\subsection{Determinação do EHL da Manteiga de Manga}

Foi determinado o EHL (equilíbrio hidrófilo-lipófilo) da manteiga de manga de acordo com Prista (1990), adaptado por Zanin \& Miguel (2004). Consistiu na preparação de emulsões seriadas da manteiga de manga obtidas através de um par de emulgentes de EHL conhecido, misturados em proporções variáveis, de modo a originarem valores definidos e escalonados de EHL. Os tensoativos utilizados foram monoleato de sorbitano (Span 80, EHL 4,7) e sesquioleato de sorbitano (Tween 80, de EHL 15,0).

Para determinar o EHL, teve-se como base a emulsão que apresentou maior estabilidade, ou seja, não apresentou separação de fases nem aspecto grumoso.

\subsection{Obtenção da Emulsão}

Para possibilitar a comparação e confirmar o efeito hidratante do ativo principal, foram preparadas três tipos de emulsões. Primeiramente a emulsão não 
iônica (emulsão base), posteriormente a emulsão base com o óleo de abacate e finalmente a emulsão base com óleo de abacate e manteiga de manga, conforme descrição na tabela 1.

TABELA 1: Componentes da formulação

\begin{tabular}{c|l|c}
\hline FASE & \multicolumn{1}{|c}{ COMPONENTES } & $\%$ \\
\hline \multirow{5}{*}{ Oleosa } & Crodabase & 12 \\
& Älcool Cetoestearílico Etoxilado & 4 \\
& Monoestearato de Glicerila & 3 \\
& Monoleato de Sorbitano & 2.5 \\
& Butilhidroxitolueno & 0.05 \\
& Óleo de Abacate & 1 \\
& Manteiga de Manga & 2 \\
& Propilparabeno & 0.2 \\
\hline \multirow{3}{*}{ Aquosa } & Sesquioleato de Sorbitano & 2.5 \\
& Metilparabeno & 0.2 \\
& EDTA-Na & 0.1 \\
& Água & qsp 100 \\
\hline
\end{tabular}

\subsection{Determinação do EHL Requerido da Emulsão}

Somou-se as porcentagens dos componentes da fase oleosa a ser emulsionada ( $\Sigma=10 \%$ ) e dividiu-se a porcentagem individual de cada componente pelo peso total da fase oleosa para encontrar o fator de contribuição de cada um. Para determinar o EHL da formulação multiplicou-se este fator de contribuição pelo EHL requerido de cada componente. O somatório dos valores é o EHL da mistura, conforme mostra a tabela 2 .

TABELA2: Determinação do EHL da Formulação

\begin{tabular}{l|c}
\hline COMPONENTES & $\%$ \\
Óleo Abacate $(E H L=6,00)$ & 0.6 \\
Manteiga de Manga $(E H L=9,85)$ & 1.97 \\
Monoestearato de Glicerila $(E H L=3,50)$ & 1.05 \\
Alcool Cetoestearilico $(E H L=15,00)$ & 6 \\
\hline & $\Sigma=9,62$ \\
\hline
\end{tabular}


Para calcular a quantidade requerida de cada emulgente ( $A$ e $B$ ) utilizado na formulação, utilizou-se a fórmula abaixo:

$$
\% A=100(X-E H L B) /(E H L A-E H L B)
$$

$\% B=100-\% A$

O par de emulsificantes utilizados foi constituído de Span 80 (EHL 4,3) e Tween 80 (EHL 15,0). Como o total de emulsionantes na formulação corresponde a $5 \mathrm{~g}$, a quantidade utilizada de Tween 80 foi de 2,486g e de Span 80 foi de $2,514 \mathrm{~g}$.

\subsection{Controle de Qualidade do Produto Acabado}

Para avaliar a estabilidade acelerada, o produto acabado foi submetido à estresse mecânico. A amostra foi colocada em tubos de ensaio e centrifugada por 30 minutos à 5000 rpm. Verificou-se, a olho nu, possível modificação aparente no aspecto das emulsões, assim como a separação das fases. A amostra também foi submetida à ciclos térmicos acondicionando-a em pote de vidro transparente, aonde manteve-se por $24 \mathrm{~h}$ sob temperatura de $45^{\circ} \mathrm{C}$, seguido por $24 \mathrm{~h}$ em temperatura ambiente e outras $24 \mathrm{~h}$ sob refrigeração à $5^{\circ} \mathrm{C}$. Repetiu-se o ciclo durante 30 dias. Paralelamente aos ciclos térmicos, acondicionou-se uma amostra do creme pelo período de 8 dias na geladeira à $5{ }^{\circ} \mathrm{C}, 8$ dias na estufa à $50^{\circ} \mathrm{C}$ e 8 dias à luz solar em recipiente de vidro, realizando 3 repetições. Para avaliar os resultados foram realizadas análises físicas como verificação do $\mathrm{pH}$ e das características organolépticas.

Na verificação das características organolépticas das emulões, as qualidades desejáveis foram homogeneidade, brilho, macio, fino e opacidade. A presença de bolhas de ar é considerada um defeito sério em qualquer produto e também foi levado em consideração (Sampaio, 1995).

Realizou-se análise reológica da formulação em viscosímetro de rotação absoluta para determinação da viscosidade, comportamento de fluxo e aplicação. $O$ equipamento utilizado foi um Haake Viscotester, Vt 550, acoplado a um banho Haake $\mathrm{K} 10$ a $25^{\circ} \mathrm{C}$, sensor PK1 (1 grau).

Para verificação da espalhabilidade, aplicou-se o método do dispositivo para determinação in vitro (Knorst, 1991; Alves, 1999), metodologia esta revisada por Zanin \& Miguel (2004), sendo que os resultados correspondem à relação entre a área de espalhamento conseguido com a aplicação de esforço sobre o produto e o esforço limite.

As emulsões desenvolvidas foram submetidas à avaliação sensorial em voluntários com testes de impressão visual, sensorial olfativo, sensorial de espalhabilidade, sensorial de viscosidade, sensorial de umectância, sensorial de emoliência e sensorial de oleosidade. Participaram deste teste dez voluntários que possuíam pele com aspecto saudável, íntegra e sem qualquer patologia. Baseando-se

Visão Acadêmica, Curitiba, v.13, n.1, Jan. - Mar./2012 - ISSN 1518-5192 
nas normas éticas de testes sensoriais descritas no Centro Europeu de Validação de Métodos Alternativos - ECVAM (Guy et al, 1996), foi desenvolvido um protocolo experimental de teste sensorial aplicado a voluntários.

\section{RESULTADOS E DISCUSSÕES}

\subsection{Determinação do EHL da manteiga de manga}

O EHL da manteiga de manga ficou compreendido entre 8,82 a 10,88, ou seja, emulsões 4, 5 e 6, pelos resultados descritos na tabela 3. As fotos da microscopia (figura 1) revelam em qual emulsão os glóbulos dispersos estão mais homogêneos em diâmetro, agregação e distribuição.

TABELA 3: EHL das Emulsões Preparadas

\begin{tabular}{c|c|c|c}
\hline EMULSÃO & EHL & ASPECTO & RESULTADO \\
\hline 1 & 5,73 & Separação de Fases & Ruim \\
\hline 2 & 6,76 & $\begin{array}{c}\text { Separação de Fases } \\
\text { Ruim }\end{array}$ & Bom \\
\hline 3 & 7,79 & $\begin{array}{c}\text { Relativa homogeneidade } \\
\text { (poucos grumos) }\end{array}$ & Muito Bom \\
\hline 4 & 8,82 & Homogêneo & Excelente \\
\hline 5 & 9,85 & Homogêneo & Muito Bom \\
\hline 6 & 10,88 & Homogêneo & Ruim \\
\hline 7 & 11,91 & Separação de Fases &
\end{tabular}

FIGURA 1: Microscopia para Determinação do EHL da Manteiga de Manga

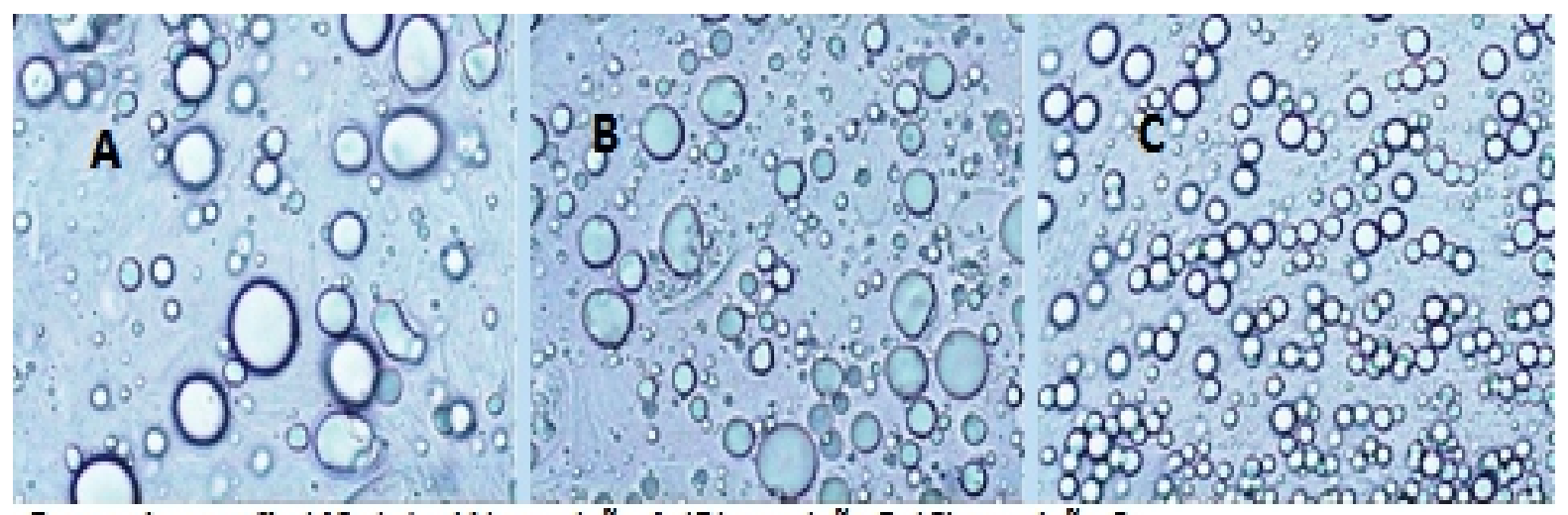

Foto-micrografia $(40 \mu)$ da: (A) emulsão 4; (B) emulsão 5; (C) emulsão 6. 
A emulsão 4 (Figura 1-A) apresentou nítida formação de glóbulos com diversos tamanhos, o que torna possível os menores agregarem-se aos maiores, desestabilizando o sistema mais rapidamente que na emulsão 5 (Figura 1 - B), onde a distribuição de tamanho dos glóbulos é mais homogênea. A emulsão 6 (Figura 1 - C) mostrou glóbulos bem formados e homogêneos, porém sem uniformidade em toda a lâmina.

Os resultados do teste de espalhabilidade das emulsões 4,5 e 6 foram respectivamente: $1451,46 \mathrm{~mm}^{2}, 1287,59 \mathrm{~mm}^{2}$ e $1045,81 \mathrm{~mm}^{2}$.

Conforme as microfotografias, as emulsões mais homogêneas foram 5 e 6 , no entanto, a melhor espalhabilidade ocorreu na emulsão 4 e 5, respectivamente. Escolheu-se então, a emulsão 5 como a ideal, já que apresentou bons resultados em ambos os testes analisados. Portanto, o EHL da manteiga de manga, equivale à 9,85 , conforme Tabela 3. A determinação deste valor teve como objetivo desenvolver um produto de melhor qualidade, com bom aspecto, brilho, espalhabilidade e principalmente estável, possibilitando a escolha de um sistema de tensoativos adequados para formulação.

\subsection{Obtenção da Emulsão}

Com a determinação do EHL da manteiga de manga, escolheu-se os ésteres de sorbitano pois costumam ser muito eficazes como emulsificantes água em óleo. Os ésteres de oleolato, tais como o monoleato de sorbitano e o sesquioleato de sorbitano ofereceram eficácia e estabilidade à formulação. Estes, associados à uma base autoemulsionante, resultaram em um produto com as qualidades desejadas e préestabelecidas para um creme hidratante.

O EHL requerido da mistura foi estabelecido baseado no valor determinado para o EHL da manteiga de manga 9,85, o que possibilitou atender as características subjetivas propostas para o produto final, como: brilho intenso, maciez, ausência de branqueamento, toque agradável e não pegajosidade.

\subsection{Controle Qualidade do Produto Acabado}

Nos testes de estabilidade acelerada a emulsão apresentou aspectos de homogeneidade, brilho, macio, fino e opacidade de acordo com as qualidades desejáveis e pré-estabelecidas para os produtos. No teste da centrifugação a amostra mostrou-se integra após tal estresse e o comportamento do creme após a permanência de 8 dias em exposição solar, em temperatura de $5^{\circ} \mathrm{C}$ e em temperatura de $50^{\circ} \mathrm{C}$; e após os ciclos térmicos, 24 horas em cada, por 30 dias, definiu tal produto como estável.

O resultado microscópico apresentado está amparado nas foto-micrografias 
em aumento de 40 X 10 das emulsões, como mostra a Figura 2.

FIGURA2: Microscopia para determinação do EHL da Emulsão

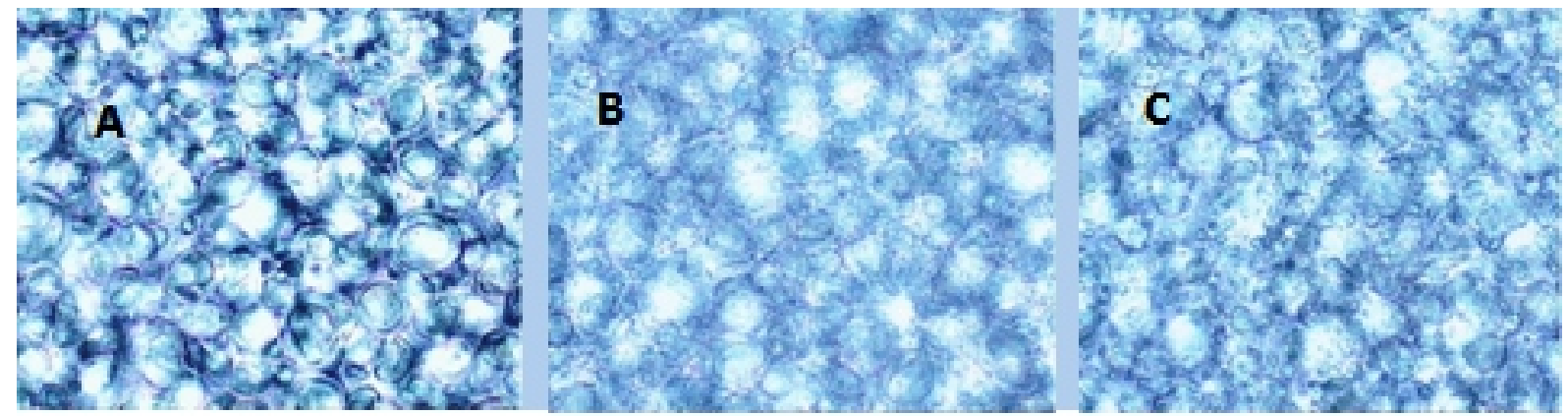

Foto-micrografia $(40 \mu)$ da: (A) emulsão base; (B) emulsão base com óleo de abacate; (C) emulsão base com óleo de abacate e manteiga de manga.

Avaliando-se a reologia pela Figura 3, pode-se observar no espectro mecânico que é necessária a aplicação de uma tensão mínima para que o fluxo viscoso se inicie (94,3 Pa). No gráfico, verificamos que a curva não tem origem no zero. Este comportamento definido como plástico é caracterizado por uma tensão inicial para o fluxo viscoso além de uma redução da viscosidade em função do aumento da taxa de cisalhamento. Pode-se observar na Figura 4 que esta redução de viscosidade foi apresentada pela amostra em análise, caracterizando novamente este material como um plástico.

O índice de fluxo apresentado pelo material em questão foi de 0,54 , indicando uma intensa dependência da viscosidade e da taxa de cisalhamento, as quais são inversamente proporcionais. Desta forma, durante a aplicação do produto, e com aumento da taxa de cisalhamento durante a aplicação, pode-se observar uma redução de viscosidade, gerando uma maior capacidade de espalhabilidade do produto.

FIGURA 3: Curva de Fluxo

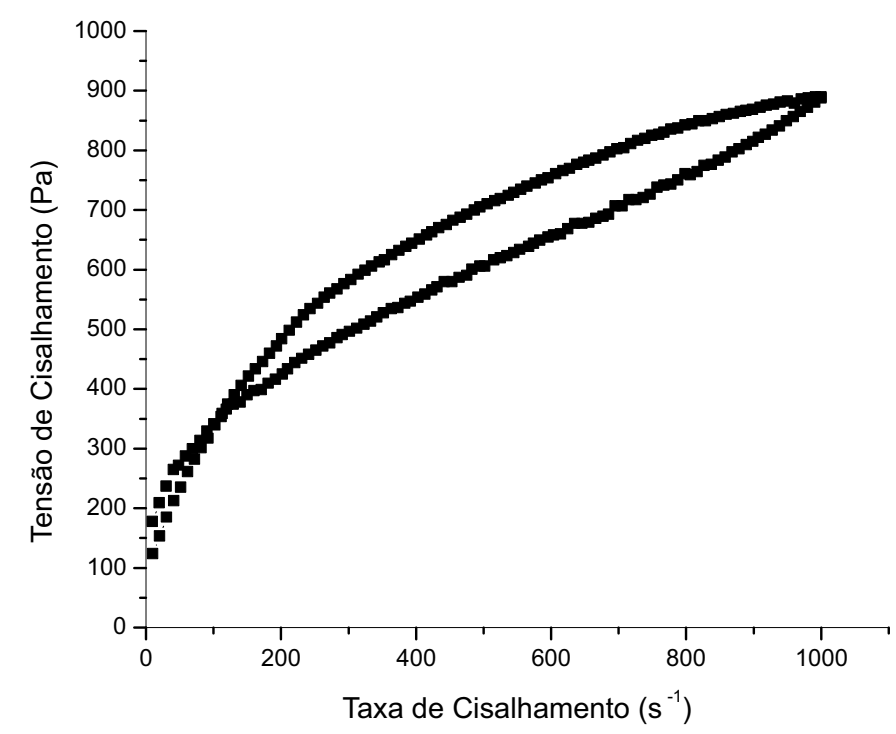


FIGURA 4: Curva de Viscosidade

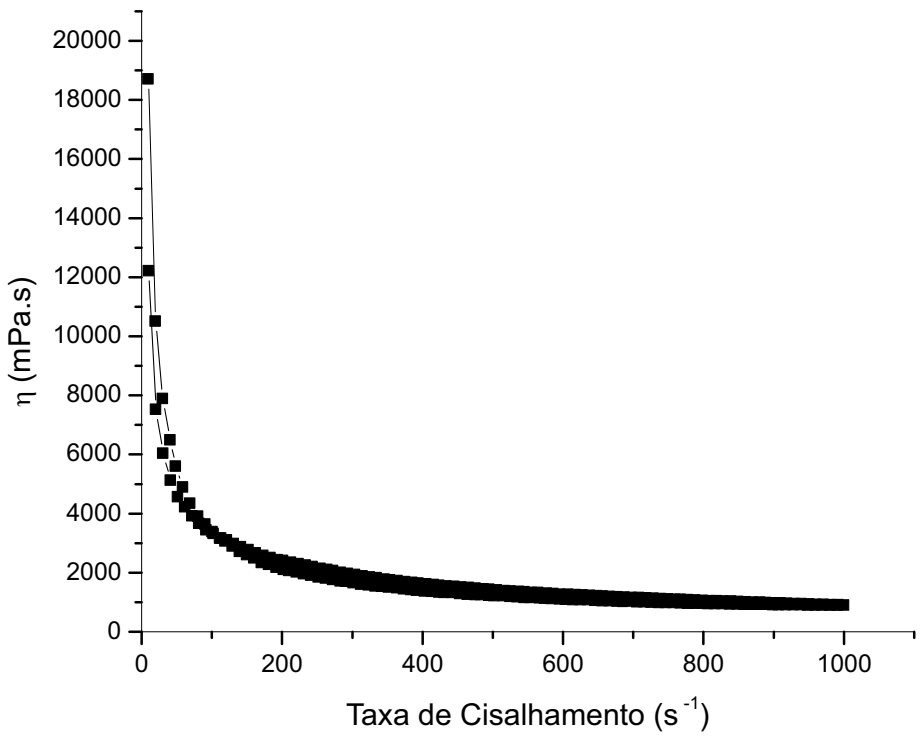

A Tabela 4 mostra os resultados de espalhabilidade e confirma o poder de aumentar a mesma no creme quando em presença do emoliente óleo de abacate, e posteriormente, em presença da manteiga de manga, representado por um valor ainda maior.

TABELA 4: Espalhabilidade das Emulsões

\begin{tabular}{l|c}
\hline EMULSÃO & $\begin{array}{c}\text { ESPALHABILIDADE } \\
\left(\mathbf{m m}^{\mathbf{2}}\right)\end{array}$ \\
\hline Base & 2094.97 \\
Base + Óleo de Abacate & 2121,00 \\
Base + Óleo de Abacate + Manteiga de Manga & 2231,77 \\
\hline
\end{tabular}

Para comparação de eficácia, foi testada primeiramente a emulsão base sem o óleo de abacate e a manteiga de manga (creme A), depois esta base acrescida com o emoliente óleo de abacate (creme B), e finalmente o produto final, com óleo de abacate e com o ativo principal, a manteiga de manga (creme $\mathrm{C}$ ). O óleo de abacate foi retirado da base (creme A) para não permitir dúvidas de que o potencial hidratante da emulsão é proveniente da manteiga de manga.

O Quadro 1 divulga os resultados da avaliação sensorial, conforme protocolo aplicado e realizado pelos 10 voluntários. Para entendimento deste método de tabulação, cada coluna possui uma sub-tabela dividida em 10 quadrículos, representando os voluntários, com notas de 1-3, atribuídas as respostas do instrumento de avaliação respondido pelos mesmos. 
QUADRO 1: Resultados da Avaliação Sensorial

\begin{tabular}{|c|c|c|c|c|c|c|c|c|c|c|c|c|c|c|c|c|c|c|c|c|c|c|c|c|c|c|c|c|c|c|c|}
\hline \multirow{4}{*}{$\begin{array}{c}\text { Emulsão } \\
\text { Base }\end{array}$} & \multicolumn{31}{|c|}{ Requisitos das Emulsões Formuladas / Notas atribuídas as emulsões testadas: 1, 2, 3} \\
\hline & \multicolumn{5}{|c|}{$\begin{array}{l}\text { Impressão } \\
\text { Visual }\end{array}$} & \multicolumn{5}{|c|}{$\begin{array}{c}\text { Sensorial } \\
\text { Olfativo }\end{array}$} & \multicolumn{5}{|c|}{$\begin{array}{c}\text { Sensorial } \\
\text { Espalhabilidade }\end{array}$} & \multicolumn{5}{|c|}{$\begin{array}{l}\text { Sensoria } \\
\text { Viscosidade }\end{array}$} & \multicolumn{5}{|c|}{$\begin{array}{c}\text { Sensorial } \\
\text { Oleosidade }\end{array}$} & \multicolumn{5}{|c|}{$\begin{array}{l}\text { Potencial } \\
\text { Hidratante }\end{array}$} & \multirow{3}{*}{$\begin{array}{c}\begin{array}{c}\text { Total de } \\
\text { Pontos }\end{array} \\
157\end{array}$} \\
\hline & 3 & 2 & 3 & 2 & 2 & 3 & 3 & 3 & 3 & 3 & 2 & 3 & 3 & 3 & 2 & 3 & 3 & 3 & 3 & 3 & 3 & 3 & 3 & 3 & 3 & 2 & 1 & 2 & 2 & 3 & \\
\hline & 3 & 2 & 3 & 3 & 2 & 3 & 2 & 3 & 2 & 2 & 1 & 2 & 3 & 3 & 3 & 3 & 3 & 3 & 3 & 3 & 3 & 3 & 3 & 3 & 3 & 1 & 1 & 3 & 3 & 2 & \\
\hline \multirow{2}{*}{$\begin{array}{c}\text { Base + Óleo de } \\
\text { Abacate }\end{array}$} & 3 & 2 & 3 & 2 & 2 & 3 & 2 & 3 & 2 & 2 & 2 & 3 & 3 & 2 & 3 & 3 & 3 & 3 & 3 & 3 & 1 & 3 & 3 & 3 & 3 & 2 & 3 & 3 & 2 & 3 & \multirow{2}{*}{159} \\
\hline & 3 & 2 & 3 & 3 & 2 & 3 & 2 & 3 & 2 & 2 & 3 & 2 & 3 & 3 & 3 & 3 & 3 & 3 & 3 & 3 & 3 & 3 & 3 & 3 & 3 & 2 & 2 & 3 & 3 & 2 & \\
\hline \multirow{2}{*}{$\begin{array}{c}\text { Base + Óleo de } \\
\text { Abacate + Manteiga } \\
\text { de Manga }\end{array}$} & 3 & 2 & 3 & 2 & 2 & 3 & 2 & 3 & 2 & 2 & 2 & 3 & 3 & 2 & 3 & 3 & 3 & 3 & 3 & 3 & 1 & 3 & 1 & 3 & 1 & 3 & 3 & 3 & 3 & 3 & \multirow{2}{*}{160} \\
\hline & 3 & 2 & 3 & 3 & 2 & 3 & 2 & 3 & 2 & 2 & 3 & 2 & 3 & 3 & 3 & 3 & 3 & 3 & 3 & 3 & 3 & 3 & 3 & 3 & 3 & 3 & 3 & 3 & 3 & 3 & \\
\hline
\end{tabular}

Notas: 1 - Fora do padrão esperado. 2 - Fora ou dentro do padrão. 3 - Dentro do padrão esperado.

Analisando a pontuação total, pode-se observar que as três emulsões comparadas tiveram boa aceitação. A emulsão contendo manteiga de manga associada ao óleo de abacate obteve a pontuação maior.

O acréscimo da mnteiga de manga e do óleo de abacate não conferiu mudanças sobre o aspecto da emulsão, segundo pode-se observar no requisito impressão visual que teve como parâmetros, cor e brilho.

Segundo os voluntários, a emulsão, quando constituída de manteiga de manga em sua formulação, mostrou espalhar mais facilmente, propriedade confirmada pelo teste de espalhabilidade in vitro (Tabela 4) e no estudo reológico do produto final.

O requisito viscosidade obteve o máximo de pontuação nas três emulsões, justificando assim, a correta determinação do EHL da manteiga de manga.

Para o parâmetro oleosidade, observa-se que apenas $20 \%$ dos voluntários tiveram sensação gordurosa quando aplicado o produto final na pele.

No potencial hidratante, tendo como parâmetros proteção e maciez da pele, $100 \%$ dos voluntários detectaram diferença sensorial na presença da manteiga de manga, confirmando a eficácia do ativo. Os resultados da avaliação sensorial levaram à confecção do gráfico a seguir.

FIGURA 5: Representação Gráfica da Avaliação Sensorial

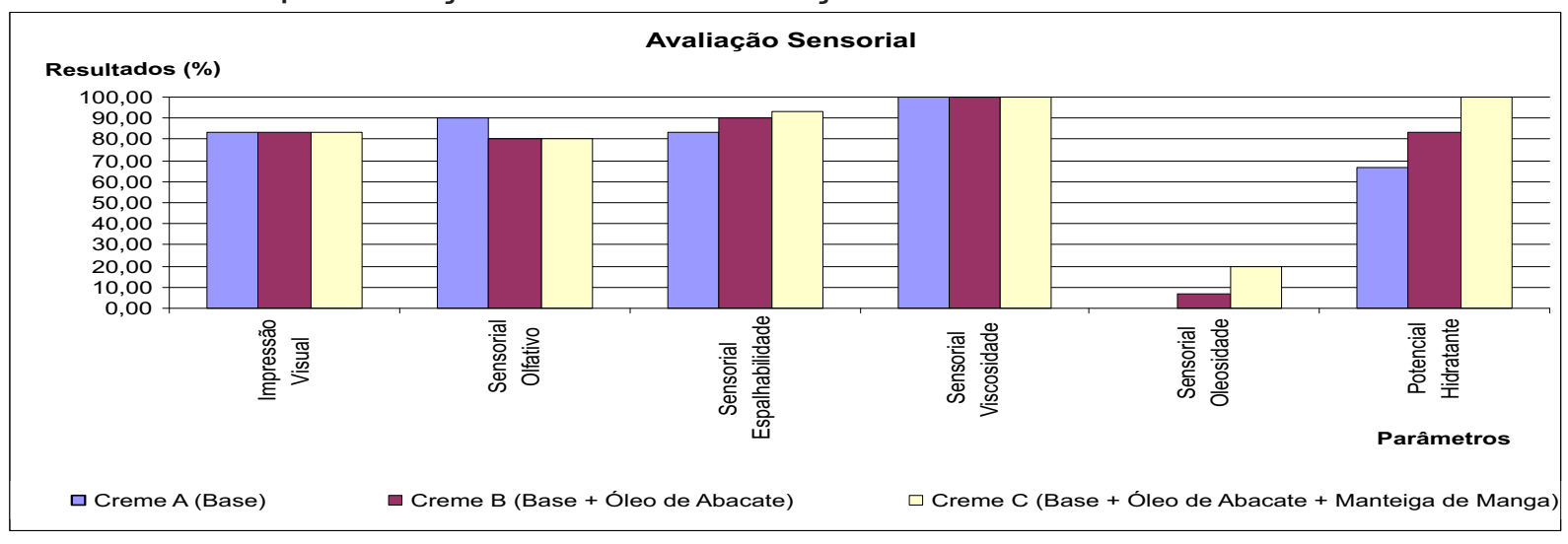




\section{CONCLUSÃO}

Após o desenvolvimento de uma emulsão dermatocosmética contendo a manteiga de manga (Mangifera indica) como ativo principal foram delineadas as seguintes conclusões:

- Quando o equilíbrio hidrófilo-lipófilo (EHL) de uma substância é desconhecido ou a literatura cita em ampla faixa de valor, torna-se importante a determinação deste valor uma vez que permite a escolha, proporção e características de tensoativos ideais para a formulação.

- As fotografias microscópicas das emulsões asseguram a estabilidade do produto final, sabendo-se que quanto menor e mais homogêneo os glóbulos, maior é a tendência à estabilidade. Além disso, a pesquisa de cada ingrediente da formulação facilita a obtenção de um produto compatível e estável.

- De acordo com a avaliação sensorial comparativa, pode-se dizer que, a formulação contendo manteiga de manga agradou àqueles que a testaram, pois atendeu as características sensoriais de espalhabilidade, oleosidade, hidratação, impressão visual e viscosidade desejáveis. A eficácia de hidratação pode ser constatada neste teste já que a maioria dos voluntários afirmou melhora deste efeito na emulsão contendo a manteiga de manga.

- Através do estudo reológico constatamos o bom espalhamento e estabilidade que o produto final apresentou. Durante a aplicação da dermoformulação desenvolvida, ocorreu um aumento da taxa de cisalhamento e uma diminuição da viscosidade, após o ponto de ruptura, característica de um comportamento plástico, isto, consequentemente, gera uma maior capacidade de espalhabilidade do produto.

- A indústria farmacêutica tem desenvolvido inúmeros ativos e bases dermatológicas de origem vegetal, no entanto, dentre as prescrições dermatocosmética que chegam às farmácias de manipulação, é comum a repetição destas variando apenas as concentrações dos ativos. Neste trabalho, a obtenção de bons resultados no desenvolvimento da emulsão, utilizando uma matéria prima pouco explorada, conclui-se a importância do estudo, uma vez que propõe o uso deste para tratamento do ressecamento da pele.

\section{REFERÊNCIAS}

ALVES, M. P.; FUNCK, J. A. B.; BITTENCOURT, C. F.; SILVA, M. A. S. Desenvolvimento e avaliação da estabilidade de bases dermatológicas não iônicas para incorporação de fármacos. Rev. Pharm. Tech., v.3, n.2, p.40-45, 1999. 
BHATTACHARYA K, SHUKLA VK. Mango butter in cosmetic formulations. Cosmet Toil 2002;117:65-70

BERGWEIN,K. Effective plant substances in cosmetic. Am. Paerf. Cosm. v.83, p.4144, may. 1968.

GUY, D.H.R. Methods for assessing percutaneous absorption. European Centre for the Validation of Alternative Methods (ECVAM) Workshop Report, Angera (Itália), v.13, p.81-106, 1996.

KNORST, M. T. Desenvolvimento tecnológico de forma farmacêutica plástica contendo extrato concentrado de Achyrocline satureioides. Lam. DC. Compositae. (Marcela) Porto Alegre, 1991. 228p. [Dissertação de Mestrado. Faculdade de Farmácia. Universidade Federal do Rio Grande do Sul].

MANDAWGADE S. D.; PATRAVALE, V.B. Formulation and Evaluation of Exotic Fat Based Cosmeceuticals for Skin Repair. Indian J Pharm Sci. 2008 Jul-Aug; 70(4): 539-542. doi: 10.4103/0250-474X.44615.

MIGUEL,M. D. ,; ZANIN S. M. W.; MIGUEL O. G.; ROZE A. O.; YAKAWA C.; OLIVEIRA A. B. O cotidiano das farmácias de manipulação. Visão Acadêmica, Curitiba, v. 3, n. 2, p. 103-108, 2002.

PRISTA, L.N. Técnica Farmacêutica e Farmácia Galênica, v.1, $4^{\circ}$ ed., Lisboa: Calouste Gulbekian, p.487-495, 1990.

PRISTA, L.N.; ALVES. A.C.; MORGANO, R.M.R. Técnica Farmacêutica e Farmácia Galênica. $3^{\circ}$ ed, Lisboa, v.2, p. 325, 326, 354, 355, 357-361, 1990.

PRISTA, L.N.; ALVES. A.C.; MORGANO, R.M.R. Técnica Farmacêutica e Farmácia Galênica. $4^{\circ}$ ed, Lisboa, v.2, p. 959-1008, 1990.

SAMPAIO, A.; Ingredientes Naturais para Cosméticos. Revista Cosmetics \&Toiletries, São Paulo, v.5, p.42-51, set./out. 1995.

ZANIN,S.M.W. et al. Determinação do Equilíbrio Hidrófilo-Lipófilo (EHL) de óleo de origem vegetal. Disponível em: http://www.visaoacademica.ufpr.br/v3n1/hidrofilo.htm.

ZANIN, S.M.W. et al. Parâmetros Físicos no Estudo da Estabilidade das Emulsões. Disponível em: http://www.visaoacademica.ufpr.br/v2n2/sandra.htm. 